\title{
Nuclear structure of the exotic mass region along the $r p$ process path
}

\author{
Yang Sun ${ }^{a}$, Michael Wiescher ${ }^{a}$, Ani Aprahamian ${ }^{a}$, Jacob Fisker ${ }^{a}$ \\ aDepartment of Physics and Joint Institute for Nuclear Astrophysics, \\ University of Notre Dame, Notre Dame, Indiana 46556, USA
}

\begin{abstract}
Isomeric states in the nuclei along the rapid proton capture process path are studied by the projected shell model. Emphasis is given to two waiting point nuclei ${ }^{68} \mathrm{Se}$ and ${ }^{72} \mathrm{Kr}$ that are characterized by shape coexistence. Energy surface calculations indicate that the ground state of these nuclei corresponds to an oblate-deformed minimum, while the lowest state at the prolate-deformed minimum can be considered as a shape isomer. The impact of these shape isomer states on isotopic abundance in x-ray bursts is studied in a multi-mass-zone x-ray burst model by assuming an upper-lower limit approach.
\end{abstract}

\section{Introduction}

It has been suggested that in x-ray binaries, nuclei are synthesized via the rapid proton capture process (rp process) [1 2], a sequence of proton captures and $\beta$ decays responsible for the burning of hydrogen into heavier elements. Recent reaction network calculations 3] have shown that the $r p$ process can extend up to the heavy Sn-Te mass region. The $r p$ process proceeds through an exotic mass region with $N \approx Z$, where the nuclei exhibit unusual structure properties. Since the detailed reaction rates depend on the nuclear structure, information on the low-lying levels of relevant nuclei is thus very important.

Depending on the shell filling, some nuclei along the $r p$ process path can have excited metastable states, or isomers [4, by analogy with chemical isomers. It is difficult for an isomeric state either to change its shape to match the states to which it is $\gamma$-decaying, or to change its spin orientation relative to an axis of symmetry. Therefore, isomer half-lives can be very long. If such states exist in nuclei along the $r p$ process path, the astrophysical significance could be that the proton-capture on long-lived isomers may increase the reaction flow, thus reducing the timescale for the $r p$ process nucleosynthesis during the cooling phase.

Coexistence of two or more stable shapes in a nucleus at comparable excitation energies has been known in nuclei with $\mathrm{A} \approx 70-80$. The nuclear shapes include, among others, prolate and oblate deformations. In an even-even nucleus, the lowest state with a prolate or an oblate shape has quantum numbers $K^{\pi}=0^{+}$. An excited $0^{+}$state may decay to the ground $0^{+}$state via an electric monopole (E0) transition. For lower excitation energies, the E0 transition is usually slow, and thus the excited $0^{+}$state becomes a "shape isomer". 


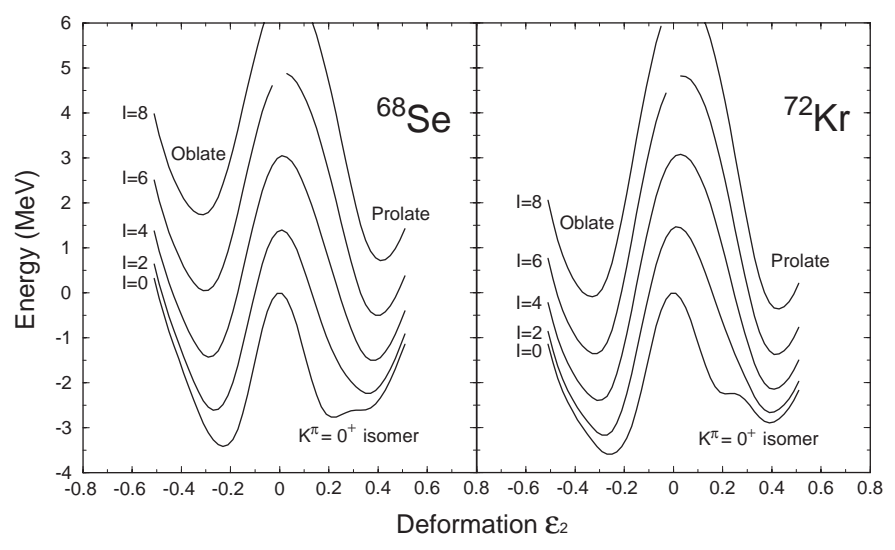

Figure 1. Energy surfaces for various spin states as a function of deformation variable $\varepsilon_{2}$.

\section{Isomers in ${ }^{68} \mathrm{Se}$ and ${ }^{72} \mathrm{Kr}$}

Nuclear structure calculations are performed in the framework of the projected shell model [5]. Fig. 1 shows calculated total energies as a function of deformation variable $\varepsilon_{2}$ for different spin states in ${ }^{68} \mathrm{Se}$ and ${ }^{72} \mathrm{Kr}$. The configuration space and the interaction strengths in the Hamiltonian are the same as those employed in the previous calculations for the same mass region [6]. Under these calculation conditions, it is found that in both nuclei, the ground state takes an oblate shape with $\varepsilon_{2} \approx-0.25$. As spin increases, the oblate minimum moves gradually to $\varepsilon_{2} \approx-0.3$. Another local minimum with a prolate shape $\left(\varepsilon_{2} \approx 0.4\right)$ is found to be $1.1 \mathrm{MeV}\left({ }^{68} \mathrm{Se}\right)$ and $0.7 \mathrm{MeV}\left({ }^{72} \mathrm{Kr}\right)$ high in excitation. Bouchez et al. [7] observed the $671 \mathrm{keV}$ shape-isomer in ${ }^{72} \mathrm{Kr}$ with half-life $\tau=38 \pm 3$ ns. The one in ${ }^{68} \mathrm{Se}$ is our prediction, awaiting experimental confirmation. Similar isomer states have also been calculated by Kaneko et al. [8].

In Fig. 2, we present the energy levels calculated by exact diagonalization, and compare them with available experimental data 9 . For ${ }^{72} \mathrm{Kr}$, with the newly confirmed $0^{+}$isomer [7] which should be the bandhead of the prolate band, the rotational band at the prolate minimum is now known. However, there have been no experimental data to compare with the predicted oblate band. In contrast, an oblate band in ${ }^{68} \mathrm{Se}$ was observed and a prolate one was also established [9], except for the missing bandhead which we predict as a shape isomer. For both nuclei, we predict low-lying $K$-isomers, indicated by bold lines. In particular, the spin-16 states are so low in excitation (much lower than the spin-16 state in the ground band) that one may consider them as an energy trap [4.

\section{Possible impact on isotopic abundance in x-ray bursts}

The recent observation of a low energy $0^{+}$shape isomer in ${ }^{72} \mathrm{Kr}$ [7] has opened new possibilities for the rp-process reaction path. A similar shape isomer has been predicted for ${ }^{68} \mathrm{Se}$ in this paper. Since the ground states of ${ }^{73} \mathrm{Rb}$ and ${ }^{69} \mathrm{Br}$ are bound with respect to these isomers, proton capture on these isomers may lead to additional strong feeding of the ${ }^{73} \mathrm{Rb}(p, \gamma){ }^{74} \mathrm{Sr}$ and ${ }^{69} \mathrm{Br}(p, \gamma){ }^{70} \mathrm{Kr}$ reactions. However, whether these branches have 

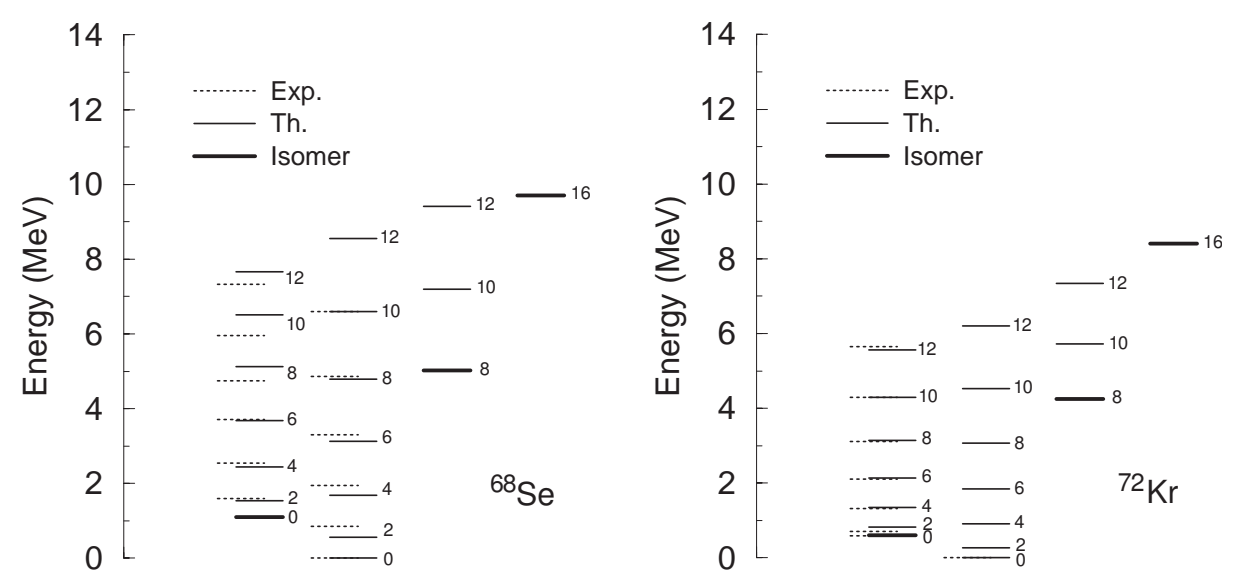

Figure 2. Energy levels for ${ }^{68} \mathrm{Se}$ and ${ }^{72} \mathrm{Kr}$.

any significance depends on the associated nuclear structure parameters, such as (1) how strong is the feeding of the isomer states?

(2) what is the lifetime of the isomer with respect to $\gamma$-decay and also to $\beta$-decay?

(3) what are the lifetimes of the proton unbound ${ }^{69} \mathrm{Br}$ and ${ }^{73} \mathrm{Rb}$ isotopes in comparison to the proton capture on these states?

Two processes can be envisaged to populate the isomeric states in appreciable abundance: either through thermal excitation of the ground state at high temperatures, or through proton capture induced $\gamma$-feeding. Thermal excitation is very efficient for feeding levels with low excitation energy since the population probability scales with $e^{-E_{i s} / k T}$. Contributions of low energy states $\left(\mathrm{E}_{x} \leq \mathrm{Q}\right)$ are negligible since proton capture on those states is balanced by inverse proton decay [2]. This is not the case for proton capture on the isomeric states. The peak temperature in the x-ray burst model employed in this work is around $1.5 \mathrm{GK}$, and the isomer states in ${ }^{68} \mathrm{Se}$ at $1.1 \mathrm{MeV}$ and in ${ }^{72} \mathrm{Kr}$ at 0.67 $\mathrm{MeV}$ are therefore only very weakly populated with the probability $\leq 0.02 \%$ and $\leq 0.5 \%$, respectively. Feeding through ${ }^{67} \mathrm{As}(p, \gamma){ }^{68} \mathrm{Se}^{*}(\mathrm{Q} \approx 3.19 \mathrm{MeV})$ and ${ }^{71} \mathrm{Br}(p, \gamma){ }^{72} \mathrm{Kr}^{*}(\mathrm{Q} \approx$ $4.1 \mathrm{MeV})$ is a more likely population mechanism. Nevertheless, a quantitative prediction of the feeding probability requires a more detailed study of the $\gamma$-decay pattern of low-spin $(\mathrm{I} \leq 3)$ states above the proton threshold in ${ }^{68} \mathrm{Se}$ and ${ }^{72} \mathrm{Kr}$, respectively.

The lifetime of the isomeric states must be sufficiently long to allow proton capture to take place. No information is presently available about the lifetime of the ${ }^{68} \mathrm{Se}^{*}$ isomer while the $55 \mathrm{~ns}$ lifetime of the isomer in ${ }^{72} \mathrm{Kr}$ [7] is rather short. Based on HauserFeshbach estimates [2] the lifetime against proton capture is in the range of $\approx 100$ ns to $10 \mu \mathrm{s}$, depending on the density in the environment. Considering the uncertainties in the present estimates a fair fraction may be leaking out of the ${ }^{68} \mathrm{Se},{ }^{72} \mathrm{Kr}$ equilibrium abundances towards higher masses.

However, the process also depends on the actual proton-decay lifetimes of ${ }^{69} \mathrm{Br}$ and ${ }^{73} \mathrm{Rb}$. Based on model dependent fragmentation cross-section predictions for these isotopes, lifetimes have been estimated to be less than $24 \mathrm{~ns}$ and $30 \mathrm{~ns}$, respectively [10]. Again, within the present systematic uncertainties this is in the possible lifetime range of proton 

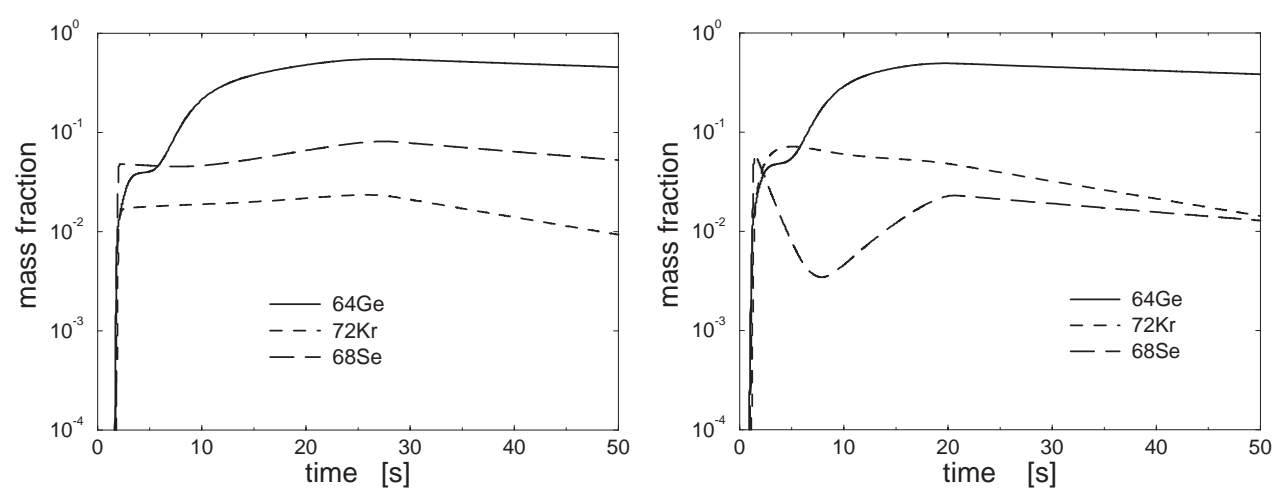

Figure 3. Mass fractions in the x-ray burst model with two extreme cases.

capture processes in high density environments.

While it is likely that equilibrium is ensued between all these configurations within the presently given experimental limits a considerable flow towards higher masses through the isomer branch cannot be excluded. Figure 3 shows the comparison between the two extreme possibilities for the reaction sequence calculated in the framework of a multimass-zone x-ray burst model [1]. The left-hand figure shows the mass fractions of ${ }^{64} \mathrm{Ge}$, ${ }^{68} \mathrm{Se}$, and ${ }^{72} \mathrm{Kr}$ as a function of time neglecting any possible isomer contribution to the flow. The right-hand figure shows the results from the same model assuming full reaction flow through the isomeric states in ${ }^{68} \mathrm{Se}$ and ${ }^{72} \mathrm{Kr}$ rather than through the respective ground states. The main differences in ${ }^{68} \mathrm{Se}$ and ${ }^{72} \mathrm{Kr}$ mass fractions are due to rapid initial depletion in the early cooling phase of the burst. This initial decline is compensated subsequently by decay feeding from the long lived ${ }^{64} \mathrm{Ge}$ abundance. The results of our model calculations shown in Fig. 3 are based on upper and lower limit assumptions about the role of the shape isomer states. The possible impact on the general nucleosynthesis of ${ }^{68} \mathrm{Se}$ and ${ }^{72} \mathrm{Kr}$ turns out to be relatively modest. These assumptions are grossly simplified. Improved calculations would require better nuclear structure data to identify more stringent limits on the associated reaction and decay rate predictions.

\section{REFERENCES}

1. L. Van Wormer et al., Astrophys. J. 432 (1994) 326.

2. H. Schatz et. al., Phys. Rep. 294 (1998) 167.

3. H. Schatz et. al., Phys. Rev. Lett. 86 (2001) 3471.

4. P. Walker and G. Dracoulis, Nature 399 (1999) 35.

5. K. Hara and Y. Sun, Int. J. Mod. Phys. E 4 (1995) 637.

6. Y. Sun, Eur. Phys. J. A 20 (2004) 133.

7. E. Bouchez et al., Phys. Rev. Lett. 90 (2003) 082502.

8. K. Kaneko, M. Hasegawa, and T. Mizusaki, Phys. Rev. C 70 (2004) 051301(R).

9. S.M. Fischer et al., Phys. Rev. Lett. 84 (2000) 4064; 87 (2001) 132501.

10. R. Pfaff et al., Phys. Rev. Lett. 53 (1996) 1753.

11. J. Fisker et al., this volume. 